\title{
The Prognostic Impact of Neutrophil Lymphocytic Ratio (NLR) on Survival of Patients with Glioblastoma Multiforme (GBM): A Retrospective Cohort Study
}

\author{
Amal Rayan ${ }^{1} \&$ Aiat M. M. Morsy ${ }^{1}$ \\ ${ }^{1}$ Clinical oncology department, Assiut faculty of medicine, Egypt \\ Correspondence: Amal Rayan, Clinical oncology department, Assiut faculty of medicine, Egypt. Tel: \\ 002-01022454482. E-mail: amal3774rayan@gmail.com
}

Received: October 1, 2017

Accepted: October 24, 2017 Online Published: October 31, 2017

doi:10.5539/cco.v6n2p69

URL: https://doi.org/10.5539/cco.v6n2p69

\begin{abstract}
Background and aim: Neoplasia related inflammation now is proved to be a factor determining the outcomes in patients with cancer including glioblastoma, we aimed to determine the prognostic value of NLR on the progression free (PFS) and overall survival (OS) for patients with GBM.

Methods: The baseline complete blood picture prior to the initiation of any corticosteroid and cancer therapy (surgery and RT) was obtained then NLR was determined and correlated with PFS and OS for patients with GBM.

Results: patients with NLR $\leq 4$ had a significantly better PFS (the median PFS $=12 \pm 1.614$ months, $\mathrm{CI}=8.836-15.164$ for those with NLR $\leq 4$ vs. a Median PFS $=6 \pm 1.239$ months, $\mathrm{CI}=3.572-8.428$ for those with $\mathrm{NLR}>4, \mathrm{P}<0.009$ ) and $\mathrm{OS}$ (the median $\mathrm{OS}=15 \pm 3.627$ months, $\mathrm{CI}=7.890-22.110$ vs. a median $\mathrm{OS}=7 \pm 1.038$ months, CI=4.966-9.034, $\mathrm{P}<0.002$ for those with NLR $\leq 4$ vs. those with NLR $>4$ respectively). And this effect of NLR was dependant on other prognostic factors.
\end{abstract}

Conclusion: NLR had a prognostic effect on PFS and OS, but it wasn't an independent factor for survival.

Keywords: glioblastoma multiforme, neutrophil-lymphocytic ratio, progression free survival, overall survival

\section{Introduction}

Gliomas are the most common primary brain tumors (Dolecek et al., 2012), the prognosis for patients with glioblastoma (GBM) is dismal with an estimated median survival of 14.6 months, and the standard treatment of GBM with maximal excision followed by temozolomide (TZM) based radiation then adjuvant TZM results in modest improvement in survival (Stupp et al., 2005).

There was sufficient evidence that immune defects were implicated in the pathogenesis of different cancers including glioblastoma (Grossman et al., 2011).

Inflammation status is now proved to be a predictor determining the outcomes in patients with cancer.

Among the markers of inflammation examined in the past years for their prognostic impact on treatment outcomes was neutrophil/lymphocytic ratio (NLR).

The neutrophil-to-lymphocyte ratio (NLR) was examined in many cancers and was found to be elevated in patients with advanced and aggressive disease (Lang et al., 2014; Xue et al., 2014; Ozdemir et al., 2014; Chang et al., 2014; Xiao et al., 2014).

Infiltration of gliomas with lymphocytes postulates that adaptive immunity maintains the tumor in a stable state (Koebel et al., 2007), which means that improved immunity is able to control and delay tumor progression.

The role of inflammatory neutrophils in controlling infections was well defined, however; they had immunosuppressive effect in cancers (Nagaraj et al., 2010), as many cancers had high blood levels of neutrophlia due to uncertain mechanisms but GM-CSF and other cytokines could be implicated in some tumors.

Bambury RM et al. Proved that patients of GBM with NLR $>4$ had a worse median overall survival at 7.5 months versus 11.2 months in patients with $\mathrm{NLR} \leq 4$ (hazard ratio $1.6,95 \%$ CI $1.00-2.52, \mathrm{p}=0.048$ ) 
(Bambury et al., 2013).

We aimed to determine the prognostic value of NLR on the progression free (PFS) and overall survival (OS) of patients with GBM.

\section{Patients and Methods}

We retrospectively performed an analysis of 58 patients with GBM who were treated with TZM based chemoradiation and adjuvant TZM with or without previous excision at clinical oncology department, Assiut university hospital during a period of two years (January 2012-December 2013).

The baseline complete blood picture was obtained prior to the initiation of any corticosteroid and cancer therapy (surgery and RT) then NLR was determined and correlated with PFS and OS for these patients.

NLR > 4 was considered a threshold of significance based on its results from other cancers.

Univariate regression test was used to determine the significance of NLR, age, sex, PS, extent of resection, response on the survival.

Multivariate regression test was used to determine the prognostic independency of NLR.

After confirmation of prognostic value of NLR on survival, patients were classified into two groups with NLR $>4$ and NLR $\leq 4$. Then survival curves for the two groups were drawn and compared.

Log rank test was used to compute survival curves for each group and compare the proportions surviving at any specific time.

PFS was defined as the time from diagnosis to demonstration of tumor progression on follow-up MRI.

OS time was defined as the time between the date of diagnosis and the date of death.

Statistical significance was defined by a $p$-value of $<0.05$, moderately significant $p$-value of $<0.01$, and highly significant $p$-value $<0.001$

\section{Results}

Characteristics of 58 patients with GBM were mentioned in table 1 including their significant impact on PFS and OS where age, KPS, extent of resection, response, and NLR had a significant effect on survival.

Table 1. Clinical and treatment characteristics of patients with GBM

\begin{tabular}{|c|c|c|c|c|c|}
\hline \multirow{2}{*}{\multicolumn{2}{|c|}{ characteristics }} & \multirow[t]{2}{*}{ NO } & \multirow[t]{2}{*}{$\%$} & \multicolumn{2}{|c|}{ Significance } \\
\hline & & & & PFS & OS \\
\hline \multirow{2}{*}{\multicolumn{2}{|c|}{ Age mean \pm SE }} & $49.72 \pm 14.04$ & & M.S. & M.S. \\
\hline & & 38 & $65 \%$ & $P<0.002$ & $\begin{array}{l}P<0.002 \\
\text { N.S. }\end{array}$ \\
\hline Sex & $\mathrm{F}$ & 20 & $35 \%$ & $P=0.703$ & $P=0.484$ \\
\hline \multirow[t]{2}{*}{ KPS } & $>70 \%$ & 19 & $32.76 \%$ & Sig. & M.S. \\
\hline & $\leq 70 \%$ & 39 & $67.24 \%$ & $P<0.022$ & $P<0.006$ \\
\hline \multicolumn{2}{|c|}{ Lateralization } & & & & N.S. \\
\hline \multicolumn{2}{|l|}{ No } & 3 & $5.2 \%$ & $P=0.343$ & $P=0.255$ \\
\hline \multicolumn{2}{|l|}{ Rt } & 19 & $32.8 \%$ & & \\
\hline \multicolumn{2}{|l|}{$\mathrm{Lt}$} & 36 & $62.1 \%$ & & \\
\hline \multicolumn{4}{|c|}{ Extent of resection } & H.S. & H.S. \\
\hline \multicolumn{2}{|c|}{ No surgery } & 14 & $24.1 \%$ & & \\
\hline \multicolumn{2}{|c|}{ Biopsy } & 14 & $24.1 \%$ & $P<0.000$ & $P<0.000$ \\
\hline \multicolumn{2}{|c|}{ Debulking } & 16 & $27.59 \%$ & & \\
\hline \multicolumn{2}{|c|}{ Subtotal excision } & 14 & $24.1 \%$ & & \\
\hline \multicolumn{2}{|c|}{ Response } & & & H.S. & H.S. \\
\hline \multicolumn{2}{|l|}{$\mathrm{CR}$} & 12 & $20.7 \%$ & & \\
\hline \multicolumn{2}{|l|}{ PR } & 15 & $25.9 \%$ & $P<0.000$ & $P<0.000$ \\
\hline \multicolumn{2}{|l|}{ SD } & 20 & $34.5 \%$ & & \\
\hline \multicolumn{2}{|l|}{ DP } & 11 & $19 \%$ & & \\
\hline \multirow[t]{2}{*}{ NLR } & $\leq 4$ & 19 & $32.8 \%$ & M.S. & M.S. \\
\hline & $>4$ & 39 & $67.2 \%$ & $P<0.009$ & $P<0.002$ \\
\hline
\end{tabular}

Sig. =significant, M.S.= moderately significant, H.S.= highly significant, NLR=neutrophil-lymphocytic ratio, CR=complete response, $\mathrm{PR}=$ partial response, $\mathrm{SD}=$ stable disease, $\mathrm{DP}=$ disease progression, $\mathrm{SE}=$ standard error, $\mathrm{KPS}=$ Karnofsky performance status. 
PFS and OS for GBM patients with NLR $\leq 4$ versus those with NLR $>4$ were mentioned in table 2 and graphed in figures 1,2 respectively.

Table 2. PFS and OS in patients with NLR $\leq 4$ versus those with NLR $>4$

\begin{tabular}{lll}
\hline NLR & PFS & OS \\
\hline$\leq 4$ & Median PFS=12 \pm 1.614 & Median OS=15 \pm 3.627 \\
& $95 \%$ CI=8.836-15.164 & $95 \%$ CI=7.890-22.110 \\
$>4$ & Median PFS=6 \pm 1.239 & Median OS=7 \pm 1.038 \\
& $95 \%$ CI=3.572-8.428 & $95 \%$ CI=4.966-9.034 \\
\hline
\end{tabular}

$\mathrm{CI}=$ confidence interval

\section{Survival Functions}

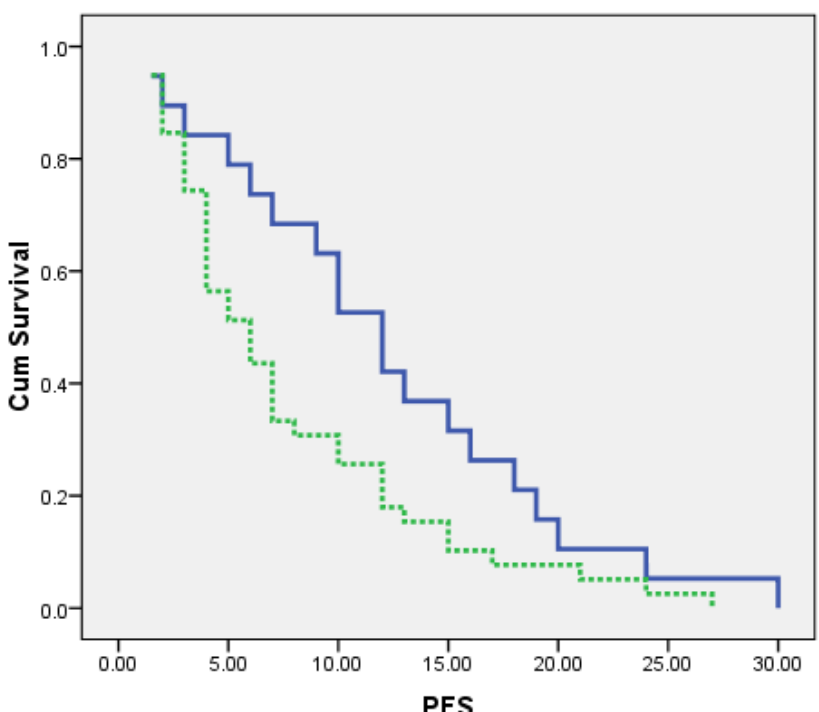

IILR

$n<4$
$-\pi>4$

Figure 1. Median PFS for patients with NLR $\leq 4$ equals $12 \pm 1.614$ (95\% CI=8.836-15.164) months, versus those with NLR $>4$ and median PFS equals $6 \pm 1.239$ (95\% CI=3.572-8.428) months, with a significant effect, $p<0.009$.

Survival Functions

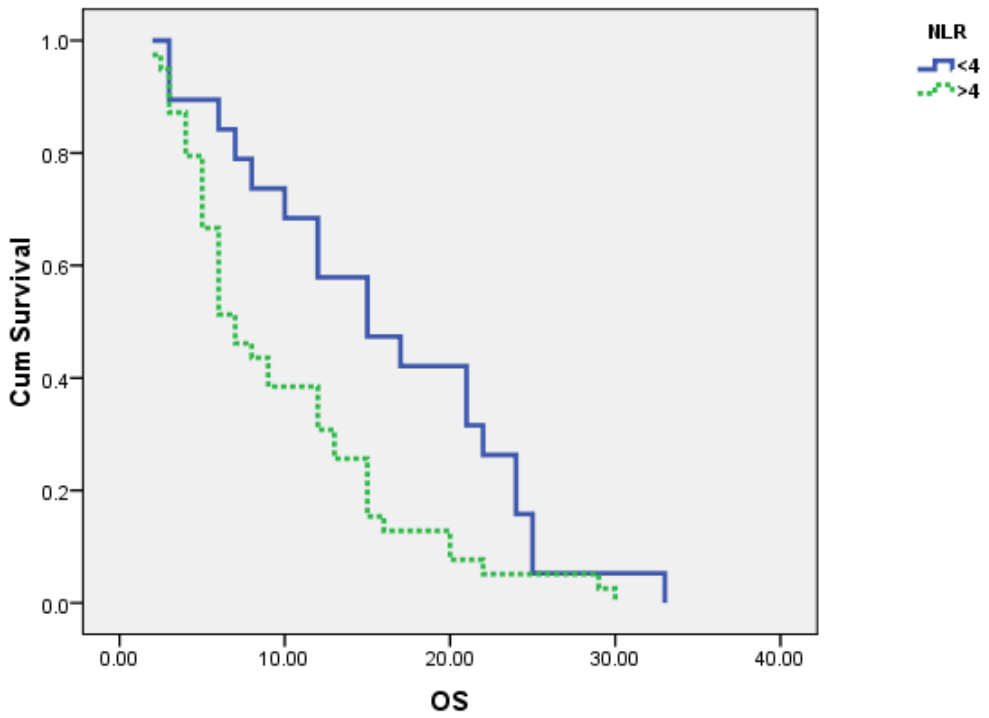

Figure 2. OS for patients with GBM in those with NLR $\leq 4$ (Median $\mathrm{OS}=15 \pm 3.627,95 \% \mathrm{CI}=7.890-22.110$ ) versus those with NLR>4 (Median OS=7士1.038, 95\% CI=4.966-9.034), with a significant effect, $p<0.002$. 
Table 3. Multivariate analysis of prognostic factors

\begin{tabular}{lllll}
\hline factor & \multicolumn{4}{c}{$\boldsymbol{P}$ value $(<\mathbf{0 . 0 5})$} \\
\cline { 2 - 5 } & PFS & \multicolumn{3}{c}{ OS } \\
\hline Response & H.S. & $\mathrm{P}<0.000$ & H.S. & $\mathrm{P}<0.000$ \\
KPS & M.S. & $\mathrm{P}<0.004$ & M.S. & $\mathrm{P}<0.001$ \\
Age & M.S. & $\mathrm{P}<0.009$ & Sig. & $\mathrm{P}<0.016$ \\
Extent of resection & S. & $\mathrm{P}<0.04$ & M.S. & $\mathrm{P}<0.006$ \\
Lateralization & N.S. & $\mathrm{P}=0.322$ & N.S. & $\mathrm{P}=0.688$ \\
NLR & N.S. & $\mathrm{P}=0.583$ & N.S. & $\mathrm{P}=0.960$ \\
\hline
\end{tabular}

KPS= Karnofsky performance status, H.S. =highly significant, M.S. =moderately significant, N.S. =non significant, Sig. = significant.

\section{Discussion}

In this study, patients with NLR $\leq 4$ had a significantly better PFS, and OS. Other demographic and clinical data with a significant effect on survival included age, KPS, extent of resection and response. But on multivariate analysis; NLR $\leq 4$ wasn't an independent factor for survival in glioblastoma patients.

A meta-analysis of nine studies including 5397 patients with nasopharyngeal carcinomas (Takenaka et al., 2017) proved that NLR greater than the cutoff values was associated with poor OS (HR 1.51, 95\% CI 1.27-1.78), disease specific survival (HR 1.44 95\% CI 1.22-1.71), PFS (HR 1.53 95\% CI 1.22-1.90), and distant metastasis free survival (HR 1.83 95\% CI 1.14-2.95).

High NLR was a poor predictor for OS with HR of 1.81 (95\% CI: 1.48-2.21; P=0.005) and relapse free survival with HR of 2.07 and $95 \% \mathrm{CI}$ : $1.65-2.6 ; \mathrm{P}=0.0849$ in a meta-analysis of 17 studies assessing the prognostic value of NLR in patients with urinary cancers (Wei et al., 2014).

Fifteen studies comprising a total of 8563 patients were included in a meta-analysis to evaluate the prognostic role of NLR among patients with breast cancer with a cutoff values ranging from 1.9-5 and found that NLR greater than the cutoff value was associated with worse OS (HR 2.56, 95\% CI=1.96-3.35; $P<0.001$ ) and disease free survival (HR 1.74, 95\% CI=1.47-2.07) (Josee-Lyne et al., 2017).

High NLR predicted a poor OS (HR 1.51; 95\% CI=1.33-1.71; P<0.001) and PFS (HR 1.33 95\% CI=1.07-1.67; $\mathrm{P}=0.012$ ) in patients with lung cancer (Qing-Tao et al., 2015).

A meta-analysis of 26 studies in primary liver cancer demonstrated that high NLR strongly predicted poor survival in these patients and high NLR was associated with vascular invasion and correlated with alpha-fetoprotein levels (Xue et al., 2014).

In a study done by Bambury et al. to evaluate the impact of NLR in 84 patients with GBM demonstrated that age over 65 years, gender, eastern cooperative oncology group performance status $\geq 2$, frontal tumor, extent of surgical resection, completion of the adjuvant chemoradiation protocol, and NLR > 4 were significantly correlated with overall survival, and the independent prognostic indicator of NLR $>4$ was maintained for poor survival in multivariate analysis.

In univariate analysis; our results were comparable to the previous studies in terms of poor survival of patients with high NLR ratio, however; in multivariate analysis, our results weren't comparable to those of Proctor et al. (Proctor et al., 2012) who studied 12,118 patients with different cancers diagnosed within 2 years and found that NLR was independently associated with survival in the studied cancers, and this could be attributed to small number of patients in this study.

In multivariate analysis our results were comparable to Mason et al. (Mason et al.,) who suggested that pretreatment NLR could not be an independent predictor of OS in gliomas.

In this study, patients with high NLR were found to be of older age, KPS $<70 \%$, no or limited surgery, and no response or even disease progression was achieved on the standard treatment protocol, so that NLR when compared to the previous prognostic factors in multivariate analysis wasn't of significant effect on survival.

\section{Conclusion}

NLR had a prognostic effect on PFS and OS, but it wasn't an independent factor for survival.

\section{References}

Dolecek, T. A., Propp, J. M., Stroup, N. E., \& Kruchko, C. (2012). CBTRUS statistical report: primary brain and 
central nervous system tumors diagnosed in the united States in 2005-2009. Neuro Oncol., 14 Suppl 5, 1-49. https://doi.org/10.1093/neuonc/nos218

Stupp, R., Mason, W. P., van den Bent, M. J., Weller, M., Fisher, B., Taphoorn, M. J., Belanger, K. et al. (2005). Radiotherapy plus concomitant and adjuvant temozolomide for glioblastoma. $N$ Engl J Med., 352, 987-96. https://doi.org/10.1056/NEJMoa043330

Grossman, S. A., Ye, X., Lesser, G., Sloan, A., Carraway, H., Desideri, S. et al. (2011). Immunosuppression in patients with high grade gliomas treated with radiation and temozolomide. Clin Cancer Res., 17, 5473-80. https://doi.org/10.1158/1078-0432.CCR-11-0774

Lang, B. H., Ng, C. P., Au, K. B., Wong, K. P., Wong, K. K., \& Wan, K. Y. (2014). Does preoperative neutrophil lymphocyte ratio predict risk of recurrence and occult central nodal metastasis in papillary thyroid carcinoma? World J Surg, 38, 2605-12. https://doi.org/10.1007/s00268-014-2630-z

Xue, T. C., Zhang, L., Xie, X. Y., Ge, N. L., Li, L. X., Zhang, B. H., Ye, S. L., \& Ren, Z. G. (2014). Prognostic significance of the neutrophil-to-lymphocyte ratio in primary liver cancer: a meta-analysis. PLoS One, 9, e96072. https://doi.org/10.1371/journal.pone.0096072

Ozdemir, Y., Akin, M. L., Sucullu, I., Balta, A. Z., \& Yucel, E. (2014). Pretreatment neutrophil/lymphocyte ratio as a prognostic aid in colorectal cancer. Asian Pac J Cancer Prev, 15, 2647-50. https://doi.org/10.7314/APJCP.2014.15.6.2647

Chang, Z., Zheng, J., Ma, Y., Zhao, J., Wang, C., \& Liu, Z. (2014). The neutrophil-to-lymphocyte ratio as a predictor for recurrence of colorectal liver metastases following radiofrequency ablation. Med Oncol, 31, 855. https://doi.org/10.1007/s12032-014-0855-1

Xiao, W. K., Chen, D., Li, S. Q., Fu, S. J., Peng, B. G., \& Liang, L. J. (2014). Prognostic significance of neutrophil-lymphocyte ratio in hepatocellular carcinoma: a meta-analysis. BMC Cancer, 14, 117. https://doi.org/10.1186/1471-2407-14-117

Koebel, C. M., Vermi, W., Swann, J. B. et al. (2007). Adaptive immunity maintains occult cancer in an equilibrium state Nature, 450, 903-907. https://doi.org/10.1038/nature06309

Nagaraj, S., Schrum, A. G., Cho, H. I., Celis, E., \& Gabrilovich, D. I. (2010). Mechanisms of cell tolerance induced by myeloid-derived suppressor cells. J. Immunol., 184, 3106-31. https://doi.org/10.4049/jimmunol.0902661

Bambury, R. M., Teo, M. Y., Power, D. G., Yusuf, A., Murray, S., Battley, J. E. et al. (2013). The association of pre-treatment neutrophil to lymphocyte ratio with overall survival in patients with glioblastoma multiforme. Journal of Neuro-oncology, 114, 149-154. https://doi.org/10.1007/s11060-013-1164-9

Takenaka, Y., Kitamura, T., Oya, R., Ashida, N., Shimizu, K., Takemura, K. et al. (2017). Prognostic role of neutrophil-lymphocyte ratio in nasopharyngeal carcinoma: A meta-analysis, Plos ONE 12(7), e0181478. https://doi.org/10.1371/journal.pone.0181478

Wei, Y., Jiang, Y-Z., \& Qian, W-H. (2014). Prognostic role of NLR in urinary cancers: A met-analysis, Plos ONE, 9(3), e92079.

Josee-Lyne, E., Danielle, D., Arnoud, T., Prakesh, S. Shan, \& Eitan, A. (2017). Prognostic role of neutrophil to lymphocytic ratio in breast cancer: a systematic review and meta-analysis. Breast Cancer Res., 19, 2.

Qing-Tao, Z., Yong, Y., Shun, X., Xiao-Peng, Z., Hui-En, W., Hua, Z., Zhi-Kang, W., Zheng, Y., \& Guo-Chen, D. (2015). Prognostic role of neutrophil to lymphocytic ratio in lung cancer: a meta-analysis including 7054 patients. Onco Targets Ther., 8, 2731-2738.

Xue, T. C., Zhang, L., Xie, X. Y., Ge, N. L., Li, L. X., Zhang, B. H. Ye, S. L., \& Ren, Z. G. (2014). Prognostic significance of the neutrophil-to-lymphocyte ratio in primary liver cancer: a meta-analysis. PLos ONE, 9 , e96072. https://doi.org/10.1371/journal.pone.0096072

Proctor, M. J., McMillan, D. C., Morrison, D. S., Fletcher, C. D., Horgan, P. G., \& Clarke, S. J. (2012). A derived neutrophil to lymphocyte ratio predicts survival in patients with cancer. Br J Cancer, 107, 695-9. https://doi.org/10.1038/bjc.2012.292

Mason, M., Maurice, C., McNamara, M. G., Tieu, M. T., Lwin, Z., Millar, B.A. et al. Neutrophil-lymphocytic ratio dynamics during concurrent chemo- radiotherapy for glioblastoma is an independent predictor for overall survival. 


\section{Copyrights}

Copyright for this article is retained by the author(s), with first publication rights granted to the journal.

This is an open-access article distributed under the terms and conditions of the Creative Commons Attribution license (http://creativecommons.org/licenses/by/4.0/). 